\title{
Effect of HDAC2/Inpp5f on neuropathic pain and cognitive function through regulating PI3K/Akt/GSK-3及 signal pathway in rats with neuropathic pain
}

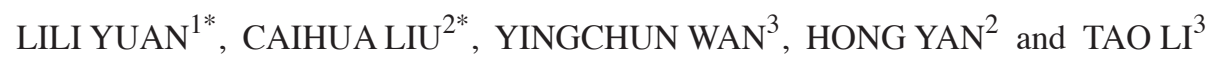 \\ ${ }^{1}$ Department of Anesthesiology, Fifth Hospital in Wuhan; ${ }^{2}$ Department of Anesthesiology, \\ The Central Hospital of Wuhan, Tongji Medical College, Huazhong University of Science and Technology, \\ Wuhan, Hubei 430050; ${ }^{3}$ Department of Endocrinology, China-Japan Union Hospital \\ of Jilin University, Changchun, Jilin 130033, P.R. China
}

Received January 29, 2019; Accepted May 7, 2019

DOI: 10.3892/etm.2019.7622

\begin{abstract}
The effect of histone deacetylase (HDAC)2/Inositol polyphosphate-5-phosphatase $\mathrm{F}$ (Inpp5f) on neuropathic pain and cognitive dysfunction through regulating $\mathrm{PI} 3 \mathrm{~K} / \mathrm{Akt} / \mathrm{GSK}-3 \beta$ signal pathway in rats with neuropathic pain was investigated. A total of 80 SPF mature male SD rats were averagely randomized into the sham operation group, the model group, the HDAC2 intervention group (group A) and the Inpp5f intervention group (group B). The rat models of neuropathic pain were established in the model group, and groups A and B. At the 15th day after modeling, rats in group A were transfected with the interference vector of HDAC2, and rats in group B were transfected with the overexpression vector of Inpp5f. Rats in the four groups were observed before modeling, after modeling/before intervention and 3 days after intervention in terms of paw thermal withdrawal latency (PWL), paw withdrawal mechanical threshold (PWT) and changes in cognitive function (Morris water maze and passive avoidance task). Then the rats were sacrificed. RT-qPCR and western blot analysis were used to detect the levels of HDAC2 mRNA, Inpp5f mRNA, phosphorylated PI3K (p-PI3K), phosphorylated AKT (p-AKT), phosphorylated GSK-3 $\beta$ (p-GSK-3 $\beta$ ) in rat brain tissue. Correlation of HDAC2 mRNA with Inpp5f mRNA expression levels was detected by Pearson's correlation analysis. Compared with the sham operation group, PWL was significantly lower while PWT was higher in the other 3 groups $(\mathrm{P}<0.05)$. Three days after intervention, PWL was significantly higher while PWT was
\end{abstract}

Correspondence to: Dr Tao Li, Department of Endocrinology, China-Japan Union Hospital of Jilin University, 126 Xiantai Avenue, Changchun, Jilin 130033, P.R. China

E-mail: tvfeq7@163.com

*Contributed equally

Key words: HDAC2, Inpp5f, PI3K/Akt/GSK-3 $\beta$, neuropathic pain, cognitive function significantly lower $(\mathrm{P}<0.05)$. Inhibiting the expression of HDAC2 or promoting the expression of Inpp5f can effectively improve cognitive function in rats $(\mathrm{P}<0.05)$. After intervention, compared with the sham operation group, rats in the other 3 groups had higher HDAC2 mRNA level and lower Inpp5f mRNA level $(\mathrm{P}<0.05)$. In conclusion, neuropathic pain can cause an increase in HDAC2 expression level and a decrease in Inpp5f expression level, and activate the PI3K/Akt/GSK-3 $\beta$ signal pathway. Inhibition of HDAC2 expression can inhibit the activation of PI3K/Akt/GSK-3 $\beta$ signal pathway through increasing Inpp5f expression, thus improving the condition and cognitive disorder of rats with neuropathic pain.

\section{Introduction}

Neuropathic pain, a complex pathological change, is stimulated or caused by the primary lesion and dysfunction of the nervous system, which is mainly related to the plasticity changes of the peripheral and central nervous systems $(1,2)$. The pathogenesis of the disease remains currently unclear. It is relatively recognized that neurological deficits lead to the sensitization of primary sensory neurons, and the enhancement of excitatory synaptic transmission in the brainstem, spinal cord and cerebral cortex, thereby resulting in chronic pain $(3,4)$. Neuropathic pain is a great challenge for clinical treatment due to its complex mechanism, and there is little specific medicine for its treatment, so it is of great significance to study the mechanism of the disease and find new therapeutic targets.

Belonging to the histone deacetylase (HDAC) family, HDAC2, widely present in eukaryotes, is important for cell proliferation and homeostasis $(5,6)$. Acetylation is irreplaceable during inflammation of chronic pain and neuronal sensitization, which opens chromatin structure, activates transcription sites and increases gene expression. Deacetylation of HDAC2 causes chromatin condensation, inhibits transcription of related genes, and results in neuropathic pain. HDACs mainly target K5, K9 and $\mathrm{K} 13$ sites on $\mathrm{H} 2 \mathrm{~A}$; K5, K12, K15 and K20 sites on H2B; K9, $\mathrm{K} 14, \mathrm{~K} 18$ and $\mathrm{K} 23$ sites on $\mathrm{H} 3$; K5, K8, K12 and K16 sites on $\mathrm{H} 4$ (7,8). Inositol polyphosphate-5-phosphatase F (Inpp5f) has the SAC phosphatase domain, so it exerts the activity of SAC 
phosphatase, inhibits the conversion of phosphatidylinosital biphosphate (PIP2) to phosphatidylinosital triphosphate (PIP3) and promotes the conversion of PIP2 to phosphatidylinosital phosphate (PIP), thus inhibiting PI3K/AKT signal pathway (9). According to a study, pregabalin effectively relieves neuropathic pain in rats with the disease, and its efficacy is related to downregulation of HDAC2 and upregulation of Inpp5f (10). Studies have also reported that the knockout of mouse HDAC2 increases Inpp5f expression, and makes the heart tolerant to the stimulation of hypertrophy, and HDAC2 is a potential target for the treatment of myocardial hypertrophy $(11,12)$. These studies indicate a close relationship among HDAC2, Inpp5f and PI3K/AKT signal pathway, which may also be the case in the occurrence and progression of neuropathic pain.

Therefore, a rat model of neuropathic pain was established in this study to explore the relationship among HDAC2, Inpp5f and PI3K/Akt/GSK-3 $\beta$ signal pathway, in order to provide an experimental basis for further understanding the mechanism of neuropathic pain in clinic.

\section{Materials and methods}

Research objects. Eighty SPF mature male SD rats were purchased from Guangdong Medical Laboratory Animal Center, fed with SPF fortified rat feeds (Jiangsu Xietong Organism Co., Ltd.). The age of the rats was 42-50 days with an average age of $46.2 \pm 3.4$ days; the weight was $216-250 \mathrm{~g}$ with an average weight of $233.6 \pm 4.8 \mathrm{~g}$; the temperature was maintained at $22 \pm 3^{\circ} \mathrm{C}$ and the humidity was $45-60 \%$. The rats were separately fed in the vivarium lighted with fluorescent lamps, free to eat and drink water, with the cage and water bottle changed once to twice weekly. The rats were randomly divided into the sham operation, the model, the HDAC2 intervention (group A) and the Inpp5f intervention (group B) groups $(n=20)$.

The study was approved by the Ethics Committee of Fifth Hospital in Wuhan (Wuhan, China).

$S D$ rat modeling. Morris water maze was carried out for one week, twice daily. The rats found the third quadrant security platform within $2 \mathrm{~min}$ and stayed on it for $45 \mathrm{sec}$, and those who failed were guided manually to complete the training. Thirty minutes later, the rat models of neuropathic pain were established in the model and intervention groups according to Chen et al (13). The rats were fixed on a larminar flow and anesthetized with an intraperitoneal injection of $10 \%$ chloral hydrate (Wuhan Yuancheng Technology Development Co., Ltd.) (300 $\mu \mathrm{g} / \mathrm{g}$ body weight). Then, skin incision was performed and the sciatic nerve was bluntly dissected. After that, 4-0 chromic catgut (Henan Zeyuan Medical Device Sales Co., Ltd.) was used to ligate the sciatic nerve trunk 3 times at intervals no more than $2 \mathrm{~mm}$, and Johnson absorbable sutures (Shanghai Hanfei Medical Instrument Co., Ltd.) to suture the incision layer by layer, with lincomycin hydrochloride $(0.2 \mathrm{ml}$, $10 \mathrm{mg} / \mathrm{ml}$ ) injected into the lateral lower limb muscles for diminishing inflammation. The ligature ensured that the nerve was compressed within 7 days while not affecting the blood transportation of the epineurium. The calf muscle of the rats slightly vibrated during ligation.
Intervention. Interference vector of HDAC2 and overexpression vector of Inpp5f, namely pCDsRed2-HDAC2-shRNA and pCDsRed2-Inpp5f-mimic, were constructed and synthesized by Takara Biotechnology Co., Ltd. At the 15th day after operation, all rats were generally anesthetized with an intraperitoneal injection of pentobarbital sodium $(50 \mathrm{mg} / \mathrm{kg})$, and received lumbar puncture at the L5-L6 intervertebral space with a puncture needle (Nanjing Jiancheng Bioengineering Institute). Rats showing lateral tail whip or cerebrospinal fluid flowing from the end of the needle tubing indicated successful puncture. A microinjector with $50 \mu \mathrm{l}$ was used to extract normal saline $(10 \mu \mathrm{g} / \mathrm{g})$, bubbles $(1 \mu \mathrm{l})$ and vectors or normal saline $(2.0 \mu \mathrm{l})$ in sequence, connected with the puncture needle to inject evenly and slowly into the spinal canal.

Observational indexes. Rats in the four groups were observed before modeling, after modeling/before intervention and 3 days after intervention in terms of paw thermal withdrawal latency (PWL), paw withdrawal mechanical threshold (PWT, measured through Von Frey filaments) and changes in cognitive function (Morris water maze and passive avoidance task). Then the rats were sacrificed. RT-qPCR and western blot analysis were used to detect the levels of HDAC2 mRNA, Inpp5f mRNA, phosphorylated PI3K (p-PI3K), phosphorylated AKT (p-AKT), phosphorylated GSK-3 $\beta$ (p-GSK-3 $\beta$ ) in rat brain tissue, Correlation of HDAC2 mRNA with Inpp5f mRNA expression levels was detected by Pearson's correlation analysis.

$P W L$. When the rats placed in the cage were quiet, the light was gathered in the middle of the bottom of the toe, and the upper limit of PWL was $20 \mathrm{sec}$ in order to avoid scalding. PWL was the time from irradiation to the rats raising the leg or escaping, the average value was obtained for 3 times with an interval of $10 \mathrm{~min}$ each time. PWL measured the day before modeling was used as a baseline value.

$P W T$. The rats' feet were stimulated through Von Frey filaments with different thresholds with the highest one of $26 \mathrm{~g}$. Rapid paw withdrawal within the stimulation time or when the filaments were removed was considered as positive. When the withdrawal was positive for 3 times within 5 consecutive tests with a certain Von Frey filament, the threshold was regarded as PWT. The interval between each experiment was $10 \mathrm{sec}$.

$R T$-qPCR. TRIzol reagent (Invitrogen; Thermo Fisher Scientific, Inc.) was used to extract total RNA in the brain tissue, with the steps carried out according to manufacturer's instructions, an ultraviolet spectrophotometer (Mettler Toledo) to analyze the concentration and purity, $3 \%$ agarose gel electrophoresis to analyze the integrity, a micro nucleic acid spectrometer to detect the purity, with A260/A280 value between 1.8 and 2.1 considered to meet the experimental requirements. After that, RT-qPCR reaction was carried out. The reverse transcription reaction system was $1.0 \mu \mathrm{l}$ of DTT $(0.1 \mathrm{M}), 2.0 \mu \mathrm{l}$ of dNTP mixture $(10 \mathrm{M}), 1.0 \mu \mathrm{l}$ of M-MLV reverse transcriptase, $2 \mu \mathrm{g}$ of total RNA, $4.0 \mu \mathrm{l}$ of $5 \mathrm{X}$ Buffer, RNAse Free $\mathrm{ddH}_{2} \mathrm{O}$ added to $20 \mu \mathrm{l}$, incubated at $75^{\circ} \mathrm{C}$ for $5 \mathrm{~min}$ and at $37^{\circ} \mathrm{C}$ for $2 \mathrm{~h}$. Then, PCR amplification was carried out, and the system was $2 \mu \mathrm{l}$ of cDNA template, $10 \mu \mathrm{l}$ of SYBR Premix Ex Taq II (2X), each $1 \mu$ l of upstream and downstream primers, double distilled water added to $20 \mu \mathrm{l}$, at $95^{\circ} \mathrm{C}$ for $3 \mathrm{~min}$, at $95^{\circ} \mathrm{C}$ for $5 \mathrm{sec}$, at $60^{\circ} \mathrm{C}$ for $34 \mathrm{sec}$, for 40 cycles. 
Table I. Primer sequences.

\begin{tabular}{lll}
\hline Variables & \multicolumn{1}{c}{ Upstream primers } & \multicolumn{1}{c}{ Downstream primers } \\
\hline HDAC2 & 5'-TGACATTGTGCTTGCTGTCC-3' & 5'-CCCTCAAGTCTCCTGTTCCA-3' \\
Inpp5f & 5'-GGAGGCCACTTGTGTAGAT-3' & 5'-GGAGGCCACTTGTGTAGAT-3' \\
GAPDH & 5'-CGGAGTCAACGGATTTGGTCGTAT-3' & 5'-AGCCTTCTCCATGGTGGTGAAGAC-3'
\end{tabular}

HDAC, histone deacetylase; Inpp5f, inositol polyphosphate-5-phosphatase F; GAPDH, glyceraldehyde-3-phosphate dehydrogenase.

Table II. Test results of PWL (sec).

\begin{tabular}{lcccccc}
\hline Variables & Sham operation group & Model group & Group A & Group B & F value & P-value \\
\hline Before modeling & $7.07 \pm 0.12$ & $7.02 \pm 0.17$ & $7.02 \pm 0.21$ & $7.02 \pm 0.23$ & 0.356 & 0.745 \\
$\begin{array}{l}\text { After modeling/ } \\
\text { before intervention }\end{array}$ & $6.96 \pm 0.22$ & $4.59 \pm 0.21^{\mathrm{a}, \mathrm{d}}$ & $4.61 \pm 0.22^{\mathrm{a}, \mathrm{d}}$ & $4.59 \pm 0.22^{\mathrm{a}, \mathrm{d}}$ & 235.647 & $<0.001$ \\
\begin{tabular}{l} 
Three days after intervention \\
\hline
\end{tabular} & $6.99 \pm 0.21$ & $3.89 \pm 0.37^{\mathrm{a}, \mathrm{d}, \mathrm{e}}$ & $6.06 \pm 0.18^{\mathrm{a}, \mathrm{b}, \mathrm{d}, \mathrm{e}}$ & $5.03 \pm 0.19^{\mathrm{a}-\mathrm{e}}$ & 571.433 & $<0.001$
\end{tabular}

${ }^{\mathrm{a}} \mathrm{P}<0.05$, compared with the sham operation group at the same time-point; ${ }^{\mathrm{b}} \mathrm{P}<0.05$, compared with the model group at the same time-point; ${ }^{\mathrm{C}} \mathrm{P}<0.05$, compared with group $\mathrm{A}$ at the same time-point; ${ }^{\mathrm{d}} \mathrm{P}<0.05$, compared with before modeling in the same group; ${ }^{\mathrm{e}} \mathrm{P}<0.05$, compared with after modeling/before intervention in the same group. PWL, paw thermal withdrawal latency.

Melting curve analysis was performed after the experiment. With glyceraldehyde-3-phosphate dehydrogenase (GAPDH) as a reaction internal reference, 3 identical wells were set for each sample, and the results were analyzed by $2^{-\Delta \Delta C q}$ (14). QuantScript RT kit was purchased from Tiangen Biotech Co., Ltd. with an item no. KR103-04, RT-qPCR detection kit from Takara Biotechnology Co., Ltd. Primer sequences were designed and synthesized by HePeng Biology (Table I).

Western blot analysis. p-PI3K, p-AKT and p-GSK-3 $\beta$ in rat brain tissue were detected. The protein concentration was measured using the BCA method (Thermo Fisher), and the concentration of the protein was adjusted to $4 \mu \mathrm{g} / \mu \mathrm{l}$. The protein was extracted from the tissue with RIPA lysate, with $50 \mathrm{ng}$ of protein loaded per lane, separated with $12 \%$ polyacrylamide gel electrophoresis with an initial voltage of $90 \mathrm{~V}$, moved to the appropriate position of the separation gel through increasing the voltage to $120 \mathrm{~V}$, transferred to the membrane [polyvinylidene fluoride (PVDF)] with a constant voltage of $100 \mathrm{~V}$ for $100 \mathrm{~min}$, and sealed at $37^{\circ} \mathrm{C}$ for $60 \mathrm{~min}$. Then, the transfer membrane was sealed in 5\% skim milk, subjected to an immune reaction, incubated with primary antibodies (p-PI3K, p-AKT and p-GSK- $3 \beta$ monoclonal antibodies; Shenyang Wanlei Bio Co., Ltd.; cat. nos. w102849, wlp001a and w103518, respectively; dilution, $1: 1,000$ ) overnight at $4^{\circ} \mathrm{C}$, washed with PBS over 5 min each time 3 times the next day, incubated with secondary antibody (goat anti-rat $\mathrm{IgG}$ polyclonal antibody; Shanghai Xinyu Bio Co., Ltd.; cat. no. xyKS016; dilution, 1:1,000) for $1 \mathrm{~h}$ at room temperature, then developed at room temperature for $1 \mathrm{~min}$ and fixed for $5 \mathrm{~min}$ with ECL luminescent reagent (Shanghai QiMing Biotechnology Co., Ltd., GJ50436). The bands scanned were statistically analyzed using Quantity One, and the relative expression level of protein = gray value of the band/gray value of the internal reference. Western blot detection kit was purchased from Shanghai Youyu
Biotechnology Co., Ltd. with cat. nos. JC-445; p-PI3K, p-AKT and p-GSK-3 $\beta$ monoclonal antibodies from Shenyang Wanlei Bio Co., Ltd. with cat. nos. w102849, wlp001a and wl03518; secondary antibody (goat anti-rat IgG) from Shanghai Xinyu Bio Co., Ltd., with cat. no. xyKS016.

Statistical analysis. SPSS19.0 [Asia Analytics (formerly SPSS China)] was used to analyze the data. Enumeration data were expressed as rate. Measurement data were expressed as mean \pm standard deviation. Analysis of variance (ANOVA) was used for comparison between groups as well as for repeated measurements for comparison at different time-points within the group and LSD test for back testing. Pearson's correlation analysis was used for the correlation of HDAC2 mRNA with Inpp5f mRNA expression levels. $\mathrm{P}<0.05$ was considered to indicate a statistically significant difference.

\section{Results}

Test results of $P W L$. There was no statistically significant difference in PWL between the four groups before modeling $(P>0.05)$, but there was a statistically significant difference after modeling/before intervention and three days after intervention $(\mathrm{P}<0.05)$. After modeling/before intervention and three days after intervention, PWL in the model group and groups $\mathrm{A}$ and $\mathrm{B}$ was lower than that in the sham operation group $(\mathrm{P}<0.05)$. After modeling/before intervention, PWL in the model group, groups A and B was not statistically different $(\mathrm{P}>0.05)$. Three days after intervention, $\mathrm{PWL}$ in groups $\mathrm{A}$ and $\mathrm{B}$ was higher than that in the model group $(\mathrm{P}<0.05)$, which in group A was higher than that in group $\mathrm{B}$ $(\mathrm{P}<0.05)$. PWL in the sham operation group was not statistically different at each time-point $(\mathrm{P}>0.05)$, which in the model group was decreased continuously $(\mathrm{P}<0.05)$. In groups $\mathrm{A}$ and $\mathrm{B}, \mathrm{PWL}$ 
Table III. Test results of PWT $(\mathrm{g})$.

\begin{tabular}{lcccccc}
\hline Variables & Sham operation group & Model group & Group A & Group B & F value & P-value \\
\hline $\begin{array}{l}\text { Before modeling } \\
\text { After modeling/ } \\
\text { before intervention }\end{array}$ & $46.66 \pm 2.57$ & $46.45 \pm 2.10$ & $46.41 \pm 2.62$ & $46.81 \pm 2.16$ & 0.124 & 0.946 \\
Three days after intervention & $45.66 \pm 2.39$ & $66.17 \pm 2.48^{\text {a,d }}$ & $65.67 \pm 3.03^{\text {a,d }}$ & $65.66 \pm 1.47^{\text {a,d }}$ & 350.961 & $<0.001$ \\
& & & & & & \\
\end{tabular}

${ }^{\mathrm{a}} \mathrm{P}<0.05$, compared with the sham operation group at the same time-point; ${ }^{\mathrm{b}} \mathrm{P}<0.05$, compared with the model group at the same time-point; ${ }^{\mathrm{c}} \mathrm{P}<0.05$, compared with group A at the same time-point; ${ }^{\mathrm{d}} \mathrm{P}<0.05$, compared with before modeling in the same group; ${ }^{\mathrm{e}} \mathrm{P}<0.05$, compared with after modeling/before intervention in the same group. PWT, paw withdrawal mechanical threshold.

Table IV. Changes in cognitive function.

\begin{tabular}{|c|c|c|c|c|c|c|}
\hline Variables & Sham operation group & Model group & Group A & Group B & F value & $\mathrm{P}$-value \\
\hline Swimming time (sec) & $80.42 \pm 16.75$ & $152.72 \pm 28.45^{\mathrm{a}}$ & $103.31 \pm 20.72^{\mathrm{a}, \mathrm{b}}$ & $124.50 \pm 24.90^{\mathrm{a}-\mathrm{c}}$ & 35.467 & $<0.001$ \\
\hline Swimming distance $(\mathrm{cm})$ & $138.62 \pm 31.27$ & $361.94 \pm 104.71^{\mathrm{a}}$ & $227.52 \pm 55.48^{a, b}$ & $256.74 \pm 57.59^{a-c}$ & 36.981 & $<0.001$ \\
\hline Number of errors (times) & $2.34 \pm 1.11$ & $9.24 \pm 3.21^{\mathrm{a}}$ & $4.79 \pm 1.87^{\mathrm{a}, \mathrm{b}}$ & $6.82 \pm 1.75^{\mathrm{a}-\mathrm{c}}$ & 38.117 & $<0.001$ \\
\hline Latent time (sec) & $13.18 \pm 5.42$ & $73.41 \pm 25.34^{\mathrm{a}}$ & $31.55 \pm 18.43^{\mathrm{a}, \mathrm{b}}$ & $52.31 \pm 17.59^{\mathrm{a}-\mathrm{c}}$ & 41.016 & $<0.001$ \\
\hline
\end{tabular}

${ }^{\mathrm{a}} \mathrm{P}<0.05$, compared with the sham operation group, ${ }^{\mathrm{b}} \mathrm{P}<0.05$, compared with the model group, ${ }^{\mathrm{C}} \mathrm{P}<0.05$, compared with group $\mathrm{A}$.

Table V. Test results of HDAC2/Inpp5f.

\begin{tabular}{|c|c|c|c|c|c|c|}
\hline Variables & Sham operation group & Model group & Group A & Group B & $\mathrm{F}$ value & P-value \\
\hline HDAC2 mRNA & $1.83 \pm 0.34$ & $6.23 \pm 1.59^{\mathrm{a}}$ & $3.12 \pm 0.83^{\mathrm{a}, \mathrm{b}}$ & $6.02 \pm 0.74^{\mathrm{a}, \mathrm{c}}$ & 97.439 & $<0.001$ \\
\hline Inpp5f mRNA & $1.41 \pm 0.28$ & $0.59 \pm 0.09^{\mathrm{a}}$ & $0.73 \pm 0.14^{\mathrm{a}, \mathrm{b}}$ & $1.15 \pm 0.12^{\mathrm{a}-\mathrm{c}}$ & 94.716 & $<0.001$ \\
\hline
\end{tabular}

${ }^{\mathrm{a}} \mathrm{P}<0.05$, compared with the sham operation group, ${ }^{\mathrm{b}} \mathrm{P}<0.05$, compared with the model group, ${ }^{\mathrm{c}} \mathrm{P}<0.05$, compared with group $\mathrm{A}$. HDAC, histone deacetylase; Inpp5f, inositol polyphosphate-5-phosphatase F.

was lower after modeling/before intervention and three days after intervention than that before modeling $(\mathrm{P}<0.05)$, which was higher three days after intervention than that after modeling/before intervention $(\mathrm{P}<0.05$; Table II).

Test results of $P W T$. There was no statistically significant difference in PWT between the four groups before modeling ( $P>0.05)$, but there was a statistically significant difference after modeling/before intervention and three days after intervention $(\mathrm{P}<0.05)$. After modeling/before intervention and three days after intervention, PWT in the model group, groups A and B was higher than that in the sham operation group $(\mathrm{P}<0.05)$. After modeling/before intervention, $\mathrm{PWT}$ in groups $\mathrm{A}$ and $\mathrm{B}$ was not different $(\mathrm{P}>0.05)$. Three days after intervention, $\mathrm{PWT}$ in groups A and B was lower than that in the model group $(\mathrm{P}<0.05)$, which in group $\mathrm{A}$ was lower than that in group $\mathrm{B}$ $(\mathrm{P}<0.05)$. PWT in the sham operation group was not different at each time-point $(\mathrm{P}>0.05)$, which in the model group was increased continuously $(\mathrm{P}<0.05)$. In groups $\mathrm{A}$ and $\mathrm{B}, \mathrm{PWT}$ was higher after modeling/before intervention and three days after intervention than that before modeling $(\mathrm{P}<0.05)$, which was lower three days after intervention than that after modeling/before intervention $(\mathrm{P}<0.05$; Table III).

Changes in cognitive function. Three days after intervention, there were statistically significant differences between the four groups in terms of swimming time, swimming distance, number of errors and latent time $(\mathrm{P}<0.05)$, which in the model group, and groups $\mathrm{A}$ and $\mathrm{B}$ were longer than those in the sham operation group $(\mathrm{P}<0.05)$, and which in groups $\mathrm{A}$ and $\mathrm{B}$ were shorter than those in the model group $(\mathrm{P}<0.05)$, and which in group A were shorter than those in group $\mathrm{B}(\mathrm{P}<0.05$; Table IV).

Test results of HDAC2/Inpp5f. Three days after intervention, there were statistically significant differences in the expression levels of HDAC2 mRNA and Inpp5f mRNA between the four groups $(\mathrm{P}<0.05)$. Compared with the sham operation group, the rats in the model group and groups A and B had higher HDAC2 mRNA expression level $(\mathrm{P}<0.05)$, but lower Inpp5f mRNA expression level $(\mathrm{P}<0.05)$. HDAC2 mRNA expression level was lower in 
Table VI. PI3K/Akt/GSK-3 $\beta$ signal pathway related proteins.

\begin{tabular}{lcccccr}
\hline Variables & Sham operation group & Model group & Group A & Group B & F value & P-value \\
\hline p-PI3K & $0.943 \pm 0.024$ & $1.463 \pm 0.135^{\mathrm{a}}$ & $1.125 \pm 0.098^{\mathrm{a}, \mathrm{b}}$ & $1.246 \pm 0.099^{\mathrm{a}-\mathrm{c}}$ & 99.689 & $<0.001$ \\
p-AKT & $1.399 \pm 0.055$ & $1.942 \pm 0.213^{\mathrm{a}}$ & $1.617 \pm 0.123^{\mathrm{a}, \mathrm{b}}$ & $1.779 \pm 0.163^{\mathrm{a}-\mathrm{c}}$ & 47.745 & $<0.001$ \\
p-GSK-3 $\beta$ & $1.109 \pm 0.072$ & $1.671 \pm 0.154^{\mathrm{a}}$ & $1.285 \pm 0.103^{\mathrm{a}, \mathrm{b}}$ & $1.375 \pm 0.081^{\mathrm{a}-\mathrm{c}}$ & 95.838 & $<0.001$ \\
\hline
\end{tabular}

${ }^{\mathrm{a}} \mathrm{P}<0.05$, compared with the sham operation group, ${ }^{\mathrm{b}} \mathrm{P}<0.05$, compared with the model group, ${ }^{\mathrm{c}} \mathrm{P}<0.05$, compared with group $\mathrm{A}$. $\mathrm{p}-\mathrm{PI} 3 \mathrm{~K}$, phosphorylated PI3K; p-AKT, phosphorylated AKT; p-GSK-3 $\beta$, phosphorylated GSK-3 $\beta$.

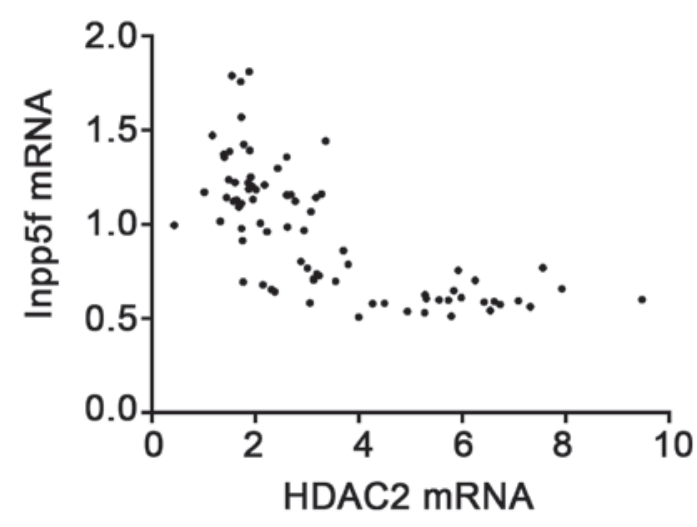

Figure 1. Correlation analysis of HDAC2 mRNA with Inpp5f mRNA expression levels. According to Pearson's correlation analysis, the expression level of HDAC2 mRNA was negatively correlated with that of Inpp5f mRNA ( $\mathrm{r}=-0.695, \mathrm{P}<0.001)$. HDAC, histone deacetylase; Inpp5f, inositol polyphosphate-5-phosphatase F.

group $\mathrm{A}$ than that in the model group and group $\mathrm{B}(\mathrm{P}<0.05)$, whereas Inpp5f mRNA expression level in groups $A$ and $B$ was higher than that in the model group $(\mathrm{P}<0.05)$. There was no statistical difference in HDAC2 mRNA expression level between the model group and group $\mathrm{B}(\mathrm{P}>0.05)$, whereas Inpp5f mRNA expression level in group $B$ was higher than that in group $\mathrm{A}(\mathrm{P}<0.05$; Table $\mathrm{V})$.

Test results of PI3K/Akt/GSK-3 $\beta$ signal pathway related proteins. There were statistically significant differences in the expression levels of p-PI3K, p-AKT and p-GSK-3 $\beta$ between the four groups $(\mathrm{P}<0.05)$, which in the model group, and groups $\mathrm{A}$ and $\mathrm{B}$ were higher than those in the sham operation group $(\mathrm{P}<0.05)$, and which in groups $\mathrm{A}$ and $\mathrm{B}$ were lower than those in the model group $(\mathrm{P}<0.05)$, and which in group $\mathrm{A}$ were lower than those in group $\mathrm{B}(\mathrm{P}<0.05$; Table VI).

Correlation analysis of HDAC2 $m R N A$ with Inpp $5 f m R N A$ expression levels. According to Pearson's correlation analysis, the expression level of HDAC2 mRNA was negatively correlated with that of Inpp5f mRNA ( $r=-0.695, P<0.001$; Fig. 1).

\section{Discussion}

Neuropathic pain is a complex pain syndrome. The incidence rate is approximately $1.5 \%$ in the world, and in China it is increasing year by year, so finding effective treatments is urgent $(15,16)$. A rat model of neuropathic pain was established in this study to explore the antagonism and mechanism of HDAC2/Inpp5f on neuropathic pain, so as to provide effective therapeutic targets and experimental bases for the prevention and treatment of neuropathic pain and cognitive dysfunction.

A rat model of neuropathic pain suffers from neuropathic pain about $24 \mathrm{~h}$ after operation for approximately 10 weeks, which is similar to characteristics of clinical neuropathic pain $(17,18)$, and meets the requirements of this experiment. PWL and PWT describe neuropathic pain in rats (19), which are used to describe the rat model in studies on neuropathic pain. In this study, compared with the sham operation group, rats in the model group had significantly lower PWL but higher PWT. Additionally, rats with neuropathic pain licked and sucked or swung the stimulated hind limbs in the air. The combination of the two indicates that the model rats had neuropathic pain, so the rat model of neuropathic pain in this experiment was successfully established. Three days after intervention in HDAC2 and Inpp5f expression, PWL was significantly higher but PWT was significantly lower compared with before intervention, indicating that inhibition of HDAC2 expression or promotion of Inpp5f expression has a good antagonistic effect on neuropathic pain in rats. In this study, inhibition of HDAC2 expression resulted in an increase in Inpp5f expression, whereas promotion of Inpp5f expression had no obvious effect on HDAC2 expression, and HDAC2 expression level was negatively correlated with Inpp5f expression level. These findings indicate that there is one-way regulation between HDAC2 and Inpp5f, and inhibition of HDAC2 expression can promote Inpp5f expression. Currently, there are few reports on the roles of HDAC 2 and Inpp $5 f$ in neuropathic pain. According to other studies, HDAC2 in LacZ mice resists cardiomyocyte hypertrophy and pressure-dependent cardiac pachynsis through increasing Inpp5f expression $(11,20)$. In addition, HDAC 2 promotes tumor development through inhibiting Inpp5f (21). These studies prove that HDAC2 has a regulatory relationship with Inpp5f, thus confirming our conclusions.

In this study, the established rat model of neuropathic pain activated PI3K/Akt/GSK-3 $\beta$ signal pathway in the brain tissue, and the expression levels of p-PI3K, p-AKT and p-GSK-3 $\beta$ were significantly higher than those in the sham operation group. Besides, after intervention in HDAC2 or Inpp5f expression, inhibition of HDAC2 expression or promotion of Inpp5f expression reduced the expression levels of p-PI3K, p-AKT and p-GSK-3 $\beta$, suggesting its role as inhibiting PI3K/Akt/GSK-3 $\beta$ signal pathway, and that inhibition of HDAC2 expression is more effective than promotion of Inpp5f expression. Based on 
the previous results, HDAC2 may promote Inpp5f expression and thus inhibit PI3K/Akt/GSK-3 $\beta$ signal pathway. However, the regulation of Inpp5f expression is not the only mechanism of HDAC2. According to a study, HDAC has a regulatory effect on PI3K/Akt/GSK-3 $\beta$ signal pathway, and inhibition of HDAC regulates polarization of microglial cells/macrophages through inhibiting GSK-3 $\beta / \mathrm{PTEN} / \mathrm{Akt}$ axis, so as to prevent white matter damage (22). HDAC2 also regulates cardiac hypertrophy response through inhibiting the activity of GSK-3 $\beta$ (11).

Neuropathic pain, abnormal pain caused by neurological deficits, usually leads to cognitive dysfunction (1-4), so improving the cognitive function of patients is also an important goal of clinical treatment. According to this study, inhibition of HDAC2 expression or promotion of Inpp5f expression improves the cognitive function of rats, but the former is more effective. In studies on the mouse model of Alzheimer's disease, knockout of HDAC2 reverses the deacetylation of histones of learning and memory genes by HDAC2, restores the structure and synaptic plasticity of neurons, and eliminates dysmnesia related to neurodegeneration $(23,24)$. There are few studies on Inpp5f and cognitive function, but according to a study, inhibition of PI3K/Akt/GSK-3 $\beta$ signal pathway prevents and treats diabetic cognitive dysfunction (25). The inhibitory effect of Inpp5f on PI3K/Akt signal pathway is clear (9), so the conclusion that Inpp5f improves rat cognitive function is credible, which will need to be proved again in future studies.

In summary, neuropathic pain can cause an increase in HDAC2 expression level and a decrease in Inpp5f expression level, and activate the PI3K/Akt/GSK-3 $\beta$ signal pathway. Inhibition of HDAC2 expression can inhibit the activation of PI3K/Akt/GSK-3 $\beta$ signal pathway through increasing Inpp5f expression, thus improving the condition and cognitive disorder of rats with neuropathic pain.

\section{Acknowledgements}

Not applicable.

\section{Funding}

No funding was received.

\section{Availability of data and materials}

The datasets used and/or analyzed during the present study are available from the corresponding author on reasonable request.

\section{Authors' contributions}

LY wrote the manuscript. CL and YW performed PCR. HY and TL were responsible for western blot analysis. LY and CL contributed to observation indexes analysis. All authors read and approved the final manuscript.

\section{Ethics approval and consent to participate}

The study was approved by the Ethics Committee of Fifth Hospital in Wuhan (Wuhan, China).

\section{Patient consent for publication}

Not applicable.

\section{Competing interests}

The authors declare that they have no competing interests.

\section{References}

1. Mu A, Weinberg E, Moulin DE and Clarke H: Pharmacologic management of chronic neuropathic pain: Review of the Canadian Pain Society consensus statement. Can Fam Physician 63: 844-852, 2017.

2. Ryan NM, Vertigan AE and Birring SS: An update and systematic review on drug therapies for the treatment of refractory chronic cough. Expert Opin Pharmacother 19: 687-711, 2018.

3. van Hecke O, Austin SK, Khan RA, Smith BH and Torrance N: Neuropathic pain in the general population: a systematic review of epidemiological studies. Pain 155: 654-662, 2014.

4. Liem L, Russo M, Huygen FJ, Van Buyten JP, Smet I, Verrills P, Cousins M, Brooker C, Levy R, Deer T, et al: One-year outcomes of spinal cord stimulation of the dorsal root ganglion in the treatment of chronic neuropathic pain. Neuromodulation 18: 41-48, discussion 48-49, 2015.

5. Zhu W, Li Z, Xiong L, Yu X, Chen X and Lin Q: FKBP3 promotes proliferation of non-small cell lung cancer cells through regulating Sp1/HDAC2/p27. Theranostics 7: 3078-3089, 2017.

6. Zimberlin CD, Lancini C, Sno R, Rosekrans SL, McLean CM, Vlaming $H$, van den Brink GR, Bots M, Medema JP and Dannenberg JH: HDAC1 and HDAC2 collectively regulate intestinal stem cell homeostasis. FASEB J 29: 2070-2080, 2015.

7. Verrills P, Sinclair C and Barnard A: A review of spinal cord stimulation systems for chronic pain. J Pain Res 9: 481-492, 2016.

8. Kami K, Taguchi S, Tajima F and Senba E: Histone acetylation in microglia contributes to exercise-induced hypoalgesia in neuropathic pain model mice. J Pain 17: 588-599, 2016.

9. Genty J, Tetsi Nomigni M, Anton F and Hanesch U: Maternal separation stress leads to resilience against neuropathic pain in adulthood. Neurobiol Stress 8: 21-32, 2017.

10. Vakkala $M$, Järvimäki $V$, Kautiainen $H$, Haanpää $M$ and Alahuhta S: Incidence and predictive factors of spinal cord stimulation treatment after lumbar spine surgery. J Pain Res 10: 2405-2411, 2017.

11. Zhu W, Trivedi CM, Zhou D, Yuan L, Lu MM and Epstein JA: Inpp5f is a polyphosphoinositide phosphatase that regulates cardiac hypertrophic responsiveness. Circ Res 105: 1240-1247, 2009.

12. Cho YK, Eom GH, Kee HJ, Kim HS, Choi WY, Nam KI, Ma JS and Kook H: Sodium valproate, a histone deacetylase inhibitor, but not captopril, prevents right ventricular hypertrophy in rats. Circ J 74: 760-770, 2010.

13. Chen XM, Xu J, Song JG, Zheng BJ and Wang XR: Electroacupuncture inhibits excessive interferon- $\gamma$ evoked up-regulation of P2X4 receptor in spinal microglia in a CCI rat model for neuropathic pain. Br J Anaesth 114: 150-157, 2015.

14. Livak KJ and Schmittgen TD: Analysis of relative gene expression data using real time quantitative PCR and the 2(-Delta Delta C(T)) method. Methods 25: 402-408, 2001.

15. Cho JH, Lee JH, Song KS and Hong JY: Neuropathic pain after spinal surgery. Asian Spine J 11: 642-652, 2017.

16. Gilron I, Baron R and Jensen T: Neuropathic pain: Principles of diagnosis and treatment. Mayo Clin Proc 90: 532-545, 2015.

17. Genda Y, Arai M, Ishikawa M, Tanaka S, Okabe T and Sakamoto A: microRNA changes in the dorsal horn of the spinal cord of rats with chronic constriction injury: a TaqMan ${ }^{\circledR}$ low density array study. Int J Mol Med 31: 129-137, 2013.

18. Chen HP, Zhou W, Kang LM, Yan H, Zhang L, Xu BH and Cai WH: Intrathecal miR-96 inhibits Nav1.3 expression and alleviates neuropathic pain in rat following chronic construction injury. Neurochem Res 39: 76-83, 2014.

19. Deer TR, Levy RM, Kramer J, Poree L, Amirdelfan K, Grigsby E, Staats P, Burton AW, Burgher AH, Obray J, et al: Dorsal root ganglion stimulation yielded higher treatment success rate for complex regional pain syndrome and causalgia at 3 and 12 months: a randomized comparative trial. Pain 158: 669-681, 2017. 
20. Kee HJ, Eom GH, Joung H, Shin S, Kim JR, Cho YK, Choe N, Sim BW, Jo D, Jeong MH, et al: Activation of histone deacetylase 2 by inducible heat shock protein 70 in cardiac hypertrophy. Circ Res 103: 1259-1269, 2008.

21. Zhu P, Martin E, Mengwasser J, Schlag P, Janssen KP and Göttlicher M: Induction of HDAC2 expression upon loss of APC in colorectal tumorigenesis. Cancer Cell 5: 455-463, 2004.

22. Wang G, Shi Y, Jiang X, Leak RK, Hu X, Wu Y, Pu H, Li WW, Tang B, Wang Y, et al: HDAC inhibition prevents white matter injury by modulating microglia/macrophage polarization through the GSK3 $3 /$ PTEN/Akt axis. Proc Natl Acad Sci USA 112: 2853-2858, 2015

23. Gräff J, Rei D, Guan JS, Wang WY, Seo J, Hennig KM, Nieland TJ, Fass DM, Kao PF, Kahn M, et al: An epigenetic blockade of cognitive functions in the neurodegenerating brain. Nature 483: 222-226, 2012.
24. Schmauss C: The roles of class I histone deacetylases (HDACs) in memory, learning, and executive cognitive functions: a review. Neurosci Biobehav Rev 83: 63-71, 2017

25. Wang $X$ and Zhao L: Calycosin ameliorates diabetes-induced cognitive impairments in rats by reducing oxidative stress via the PI3K/Akt/GSK-3 $\beta$ signaling pathway. Biochem Biophys Res Commun 473: 428-434, 2016.

This work is licensed under a Creative Commons Attribution-NonCommercial-NoDerivatives 4.0 International (CC BY-NC-ND 4.0) License. 